Le Lait (1984), 64, 418-435

\title{
Application d'un réactif de transparisation du lait au dénombrement de bactéries
}

\author{
par \\ R. KOUOMEGNE, P. BRACQUART et G. LINDEN
}

\begin{abstract}
Rés u m é
Après addition du mélange dissolvant n-butylamine, cyclohexanone et triton X-100, le lait perd sa turbidité naturelle du fait de la dissolution complète de ses constituants collö̈daux. Ce traitement permet de le soumettre directement aux méthodes optiques de détection de micro-organismes. Les mesures faites au spectromètre d'absorption moléculaire et au néphélémètre laser nous permettent de tracer respectivement des courbes de tension et de densité optique en fonction du poids sec des cellules $(\mathrm{mg} / \mathrm{ml})$ et en fonction du nombre de germes par $\mathrm{ml}$ exprimés en unités formant colonies (U.F.C./ml). Cette méthode de détection est simple ; elle permet d'évaluer rapidement le nombre de germes présents dans le lait ; elle est d'une bonne répétabilité.
\end{abstract}

Mots clés : Transparisation - Dissolution - Lait - Dénombrement - Microorganismes.

\section{S u m m a r y \\ APPLICATION OF TRANSPARISATION TO ENUMERATE MILK MICROORGANISMS}

Milk is naturully opaque since it contains fat globules and calcium phosphocaseinates. Thus the number of milk microorganisms can not be estimated by direct optical methods. In order to make milk transparent it was treated with a mixture of $n$-butylamine/cyclohexanone/triton $X-100$ in the ratio $2: 1: 1$ at $37^{\circ} \mathrm{C}$ and at $p H$ 11.3.

Laboratoire de Biochimie appliquée, Université de Nancy I, B.P. 239 - 54506 Vandouvre-Lès-Nancy cedex. 
When milk is placed in contact with this dissolving mixture, it becomes clear as a result of the total dissolution of its colloidal components. For optical measurement of the voltage at $632,8 \mathrm{~nm}$ we used a Behring Laser nephelemeter in which the light source is a HeliumNeon laser; a type 602 Perkin-Elmer molecular absorption spectrometer was used to measure optical density at $650 \mathrm{~nm}$.

As reference methods the standard cell dry weight and microorganism Petri dishes count methods were chosen. Voltage values and optical density values were obtained for every bacterial strain studied in Tris buffer $0.025 \mathrm{M}$ at $\mathrm{pH}$ 7.0. Theses values were obtained before and after treatment with the dissolving mixture and could be plotted against colony forming units (C.F.U./ml) and against cell dry weight (in $\mathrm{mg} / \mathrm{ml}$ ).

This new method is easy, repeatable and allows a rapid estimation of milk germs.

Key words : Dissolution - Enumeration - Milk - Microorganisms.

\section{INTRODUCTION}

Le lait présente une opalescence naturelle due essentiellement aux phosphocaséinates de calcium et aux globules gras. Il ne peut donc être soumis directement aux méthodes de mesures optiques ; il faut lui appliquer un traitement préliminaire.

Certains auteurs (Bosset et Blanc, 1977; Bosset et al., 1977 a et b) ont étudié la dissolution intégrale du lait au moyen de divers solvants mixtes tels les mélanges : hydroxyde de sodium/eau/n-butylamine et hydroxyde de sodium/eau/tétrahydrofurane. En utilisant ces solvants, ils ont réussi à doser les protéines totales du lait par la méthode dite du Biuret mais en diluant fortement le lait.

En se basant sur le même principe, Linden et Paquet (1981) ont mis au point un réactif dissolvant rapidement les constituants colloïdaux du lait après une faible dilution. En mélange avec ce réactif, le lait perd sa turbidité et devient parfaitement limpide. Ce réactif qui est un mélange de n-butylamine, de cyclohexanone et de triton $\mathrm{X}-100$ en présence de $\mathrm{HCl}$ dans les proportions $2 / 1 / 1$, a permis à Linden et al. (1982) de simplifier le protocole opératoire d'analyses d'activités enzymatiques dans le lait.

Suite à ces travaux, nous avons envisagé de coupler cette technique de dissolution des constituants colloïdaux du lait avec la néphélémétrie laser pour dénombrer les micro-organismes dans le lait.

La modernisation constante des méthodes industrielles en laiterie pose souvent le problème du contrôle de la qualité microbiologique 
de la matière première et du produit fini. Une méthode significative d'appréciation de cette qualité est le dénombrement ou l'évaluation de la flore aérobie ou aéro-anaérobie mésophile du produit. Les techniques usuelles permettant d'évaluer cette flore sont nombreuses mais mal adaptées aux progrès technologiques de l'industrie laitière car elles sont longues et peu sûres. En effet, la longueur du délai de réponse qui est d'au moins 24 h est un inconvénient majeur qui a poussé de nombreux auteurs tels Bossuyt (1982) Bouix et coll. (1982), Südi et coll. (1981) à se pencher sur d'autres techniques, dites modernes et rapides d'appréciation de la flore du lait telles que la mesure de l'impédance, le test Limulus...

L'objectif du présent travail est de dénombrer rapidement les micro-organismes du lait par une nouvelle méthode : la néphélémétrie laser, basée sur la dispersion de la lumière par des particules en suspension diluée (Ciret, 1980 ; Guinet et coll., 1979).

\section{MATERIEL ET METHODES}

\section{Souches bactériennes}

Les souches de bactéries utilisées sont essentiellement des souches pures congelées, conservées à $-18^{\circ} \mathrm{C}$; ce sont :

- Streptococcus thermophilus CNRZ 302.

- Lactobacillus casei Hd 13.

- Bacillus cereus Na5.

- Escherichia coli (Ga2, Ga5, Ga 12).

- Pseudomonas fluorescens Ba1.

Afin de ne pas alourdir cet article, nous ne rapporterons, en détails, que les résultats obtenus avec les souches de Streptococcus thermophilus CNRZ 302 et de Escherichia coli Ga5.

\section{Culture et récupération des cellules}

- Le milieu Tryptose-Protéose-Peptone-Yeast extract (T.P.P.Y.) liquide et stérile est ensemencé à l'aide d'une suspension de souche microbienne pure, préalablement décongelée et mise à incuber pendant $14 \mathrm{~h}$ à $15 \mathrm{~h}$ entre $30^{\circ} \mathrm{C}$ et $42^{\circ} \mathrm{C}$ selon les souches.

- Après culture, les cellules sont centrifugées à 600 tours/min pendant $10 \mathrm{~min}$ à $4^{\circ} \mathrm{C}$. Le culot est lavé avec $10 \mathrm{ml}$ de tampon Tris

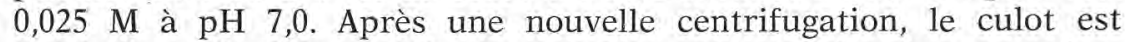
repris dans $20 \mathrm{ml}$ de ce mélange tampon ce qui constitue la suspensionmère à partir de laquelle nos essais sont effectués. 


\section{Numération}

Nous avons réalisé une série de dilutions décimales de $10^{-1}$ à $10^{-7}$ de la suspension-mère dans le liquide de dilution Tryptone-Sel (T.S.). Nous avons ensuite effectué un étalement avec $0,1 \mathrm{ml}$ de chaque dilution, à la surface du milieu Tryptose-Protéose-Peptone-Yeast extract (T.P.P.Y.) solidifié, en boîte de Pétri préalablement séchée une nuit à $30^{\circ} \mathrm{C}$.

Pour chaque dilution, 2 boîtes de Pétri sont ensemencées et le nombre de germes est donné en unités formant colonies (U.F.C.). Seules les boîtes contenant 30 à 300 colonies sont comptées.

\section{Mesures}

Pour chaque souche de cellules étudiées dans le tampon Tris $0,025 \mathrm{M}$ à $\mathrm{pH} 7,0$ avant et après addition du réactif de transparisation, les mesures sont effectuées parallèlement au néphélémètre laser Behring module 1 à $632,8 \mathrm{~nm}$ et au spectromètre d'absorption moléculaire Perkin-Elmer modèle 602 à $650 \mathrm{~nm}$.

Une observation des cellules est également faite au microscope photonique et au microscope électronique à balayage.

Dans le lait, les mesures sont faites uniquement après addition du réactif de transparisation.

Du fait de l'opacification des cuves en matière plastique par la cyclohexanone, nous avons travaillé avec des cuves en verre.

Le lait utilisé est du lait écrémé en poudre «Lesaffre », reconstitué à $10 \%$ dans de l'eau distillée et reposé $1 \mathrm{~h}$ à l'obscurité.

\section{Technique de transparisation}

Dans un tube à essais contenant $1 \mathrm{ml}$ de tampon Tris $0,025 \mathrm{M}$ à $\mathrm{pH} 7,0$, nous avons ajouté $1 \mathrm{ml}$ de suspension de cellules diluée dans le lait reconstitué ou dans le tampon Tris $0,025 \mathrm{M}$ à $\mathrm{pH} 7,0$. Après agitation, le tube est mis à incuber au bain-marie à $37^{\circ} \mathrm{C}$ pendant quelques minutes.

Nous ajoutons ensuite $2 \mathrm{ml}$ de mélange dissolvant n-butylamine/ cyclohexanone/triton X-100 (B.C.T.) à $\mathrm{pH} 11,3$; une nouvelle agitation, suivie d'une incubation de quelques secondes est nécessaire avant la lecture de la solution ainsi transparisée au néphélémètre et au spectromètre.

Avant chaque série de mesure, il est important de faire un blanc et de s'assurer que la solution transparisée est bien homogène. 


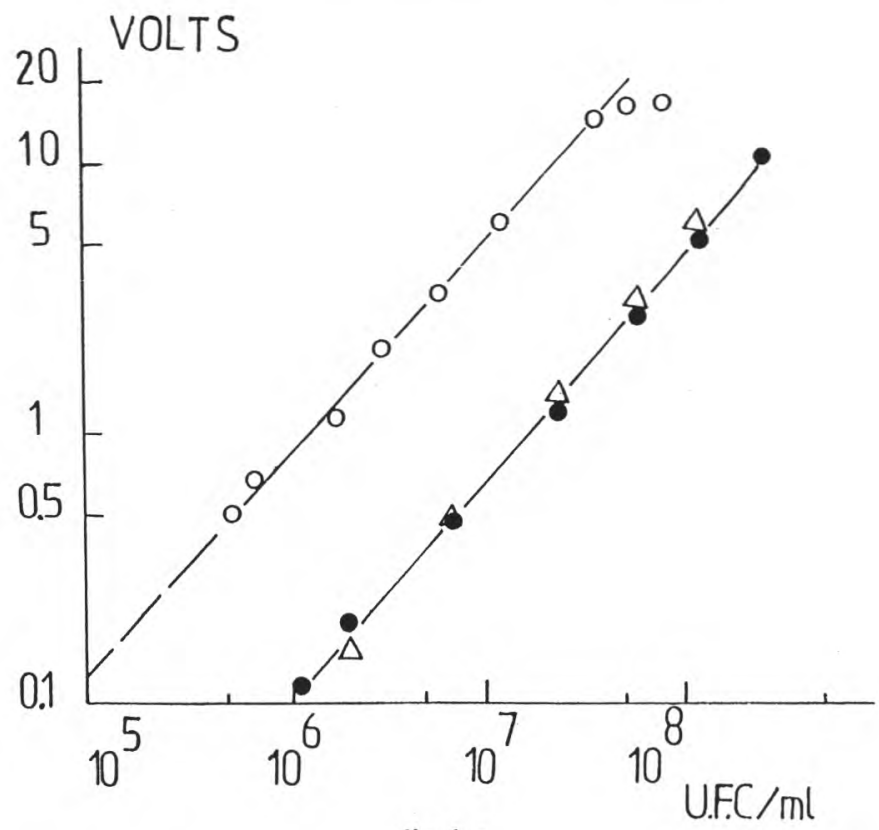

fig 1 a

Courbes d'étalonnage du néphélémètre laser pour une souche de bactérie Gram positive : $S$. thermophilus CNRZ 302 dans le tampon Tris $0,025 \mathrm{M}$ à $\mathrm{pH} 7,0$ et dans le lait.

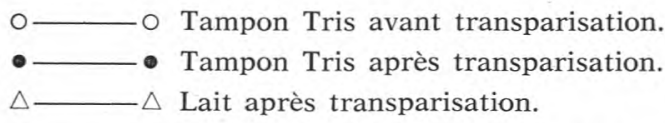

Calibration curve for the laser nephelemetre of a Gram positive bacteria specie : S. thermophilus CNRZ 302 in milk and Tris buffer $0.025 \mathrm{M}$ at pH 7.0.

$\bigcirc-0$ Tris buffer before transparisation.

- - Tris buffer after transparisation.

$\triangle \longrightarrow$ Milk after transparisation.

\section{Technique de fixation des cellules avant leur observation au microscope}

Avant leur observation au microscope, les cellules en suspension sont fixées sur les lames de verre par flambage et colorées au bleu de méthylène.

Toutes les lames observées ont été préparées à partir des dilutions des suspensions de cellules dans le tampon Tris 0,025 M à pH 7,0.

Les lames destinées au microscope électronique à balayage subissent une métallisation par vaporisation d'une suspension d'argent sous vide (Gallant, 1981). 


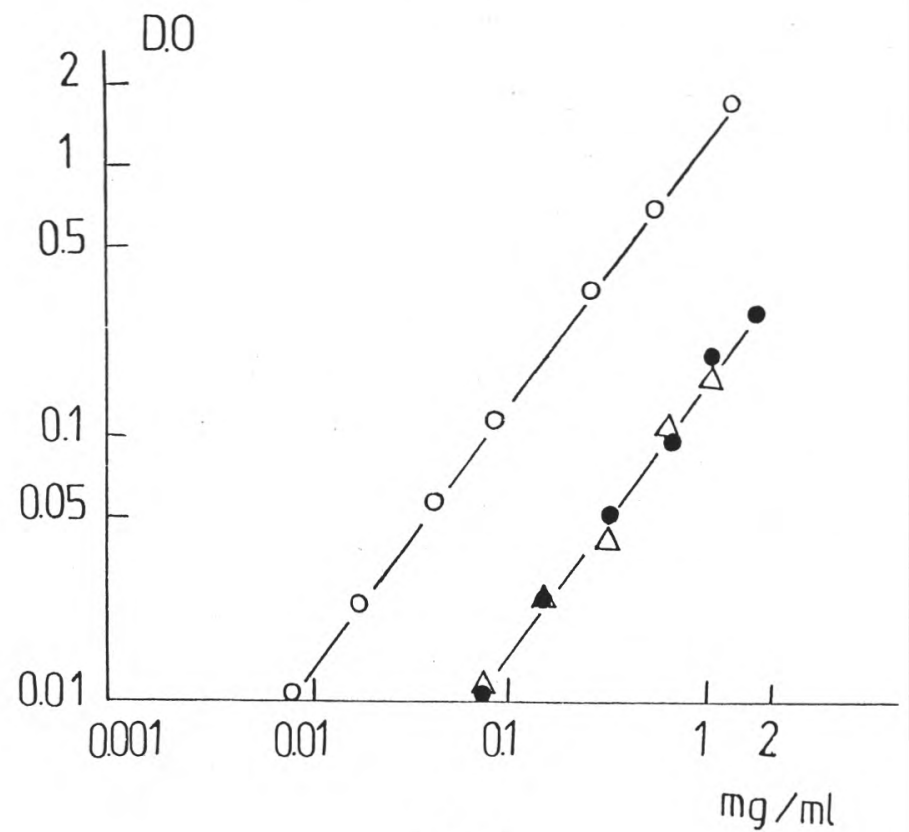

fig. $1 \quad b$

Courbes d'étalonnage du spectromètre d'absorption moléculaire pour une souche de bactérie Gram positive : S. thermophilus CNRZ 302 dans le tampon Tris $0,025 \mathrm{M}$ à $\mathrm{pH} 11,3$ et dans le lait.

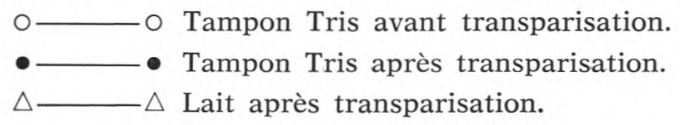

Calibration curve for the molecular absorption spectrometer of a Gram positive bacteria specie : S. thermophilus CNRZ 302 in milk and Tris buffer $0.025 \mathrm{M}$ at $\mathrm{pH}$ 11.3.

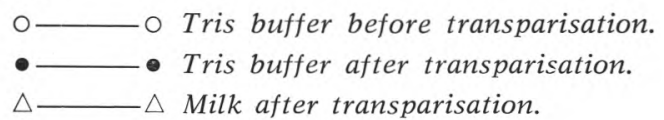

\section{RESULTATS}

\section{Essai sur les bactéries Gram positive}

Etude des différentes souches

Toutes nos mesures ont été effectuées à partir des dilutions de la suspension-mère des souches pures. 


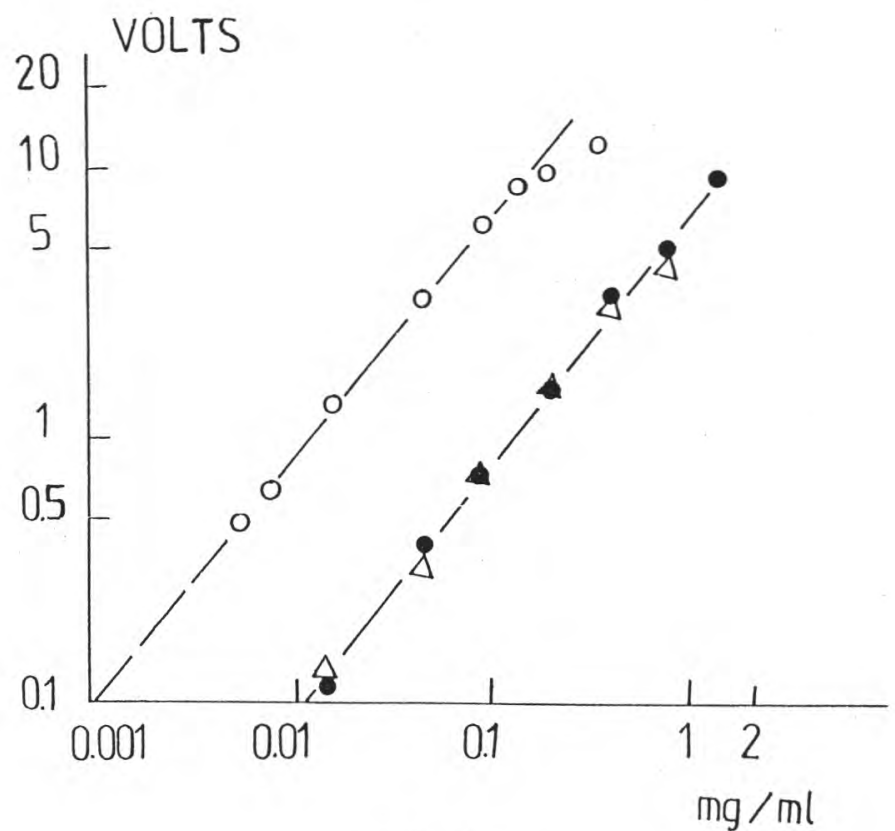

fig. $1 \mathrm{c}$

Courbes d'étalonnage du néphélémètre laser pour une souche de bactérie Gram positive : S. thermophilus CNRZ 302 dans le tampon Tris $0,025 \mathrm{M}$ à $\mathrm{pH} 7,0$ et dans le lait.

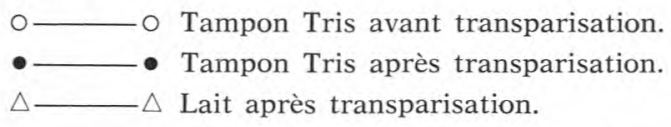

Calibration curve for the laser nephelemetre of a Gram positive bacteria specie : S. thermophilus CNRZ 302 in milk and Tris buffer $0.025 \mathrm{M}$ at pH 7.0.

○- $\mathrm{O}$ Tris buffer before transparisation.

- - Tris buffer after transparisation.

$\triangle \longrightarrow$ Milk after transparisation.

Les valeurs de tension (en volts) et de densité optique (en unités de D.O.) enregistrées respectivement au néphélémètre laser et au spectromètre d'absorption moléculaire, nous permettent de tracer des courbes de tension et de densité optique en fonction des U.F.C./ml ainsi que des courbes de tension et de densité optique en fonction du poids sec des cellules, en tenant compte des facteurs de dilutions.

Les courbes tracées ont quasiment la même allure que celles de S. thermophilus CNRZ 302 montrées ci-contre (fig. 1a, b, c, d). En comparant les courbes de tension et de densité optique, nous constatons que lorsque nous passons de la néphélémétrie (fig. 1a, 1c) à la 


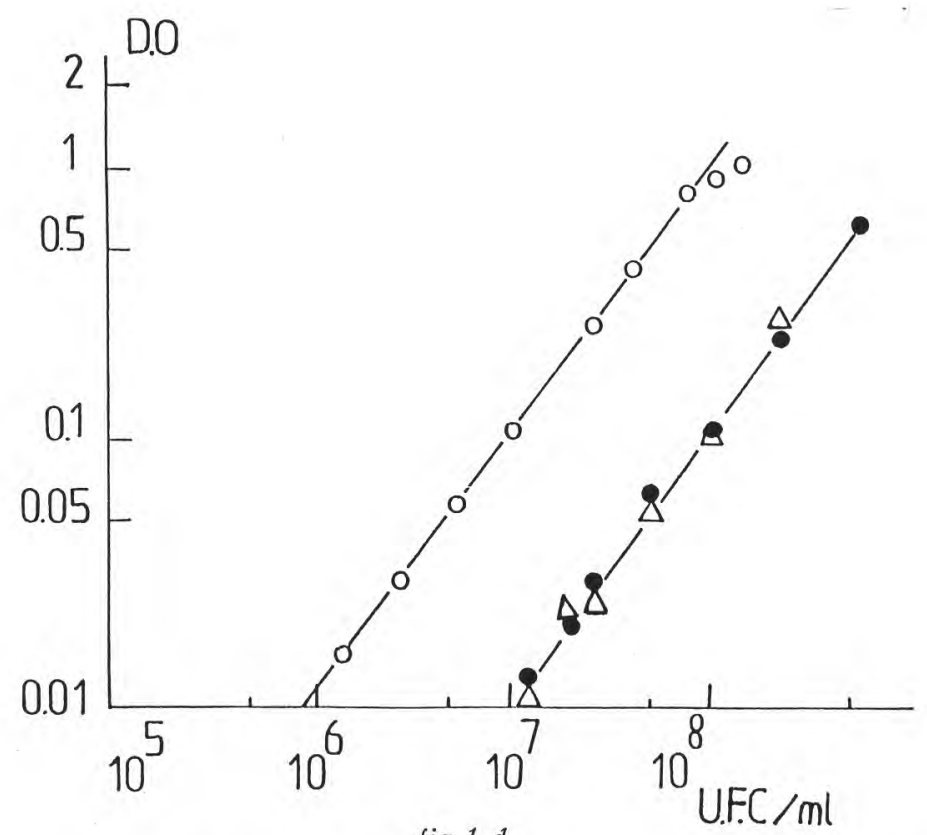

fig 1 d

Courbes d'étalonnage du spectromètre d'absorption moléculaire pour une souche de bactérie Gram positive : S. thermophilus CNRZ 302 dans le tampon Tris $0,025 \mathrm{M}$ à $\mathrm{pH} 11,3$ et dans le lait.

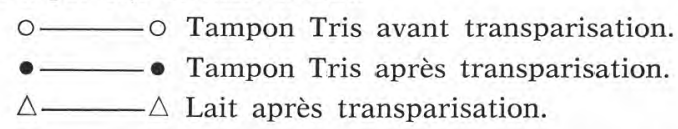

Calibration curve for the molecular absorption spectrometer of a Gram positive bacteria specie : S. thermophilus CNRZ 302 in milk and Tris buffer $0.025 \mathrm{M}$ at $p H 11.3$.

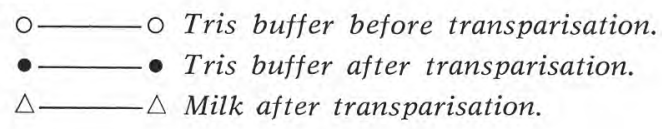

spectrométrie d'absorption moléculaire (fig. 1b, 1d), le seuil inférieur de détection diminue d'une puissance de 10 .

Pour toutes les souches Gram positive étudiées, ce seuil inférieur de détection varie avec la souche aussi bien en néphélémétrie laser qu'en spectrométrie d'absorption moléculaire lorsque nous rapportons au nombre de germes (U.F.C./ml) ; cette variation n'est plus observée si nous travaillons en poids sec des cellules.

- Après transparisation, les essais réalisés donnent lieu à des courbes d'allure identique et parallèles à celles obtenues avant trans- 


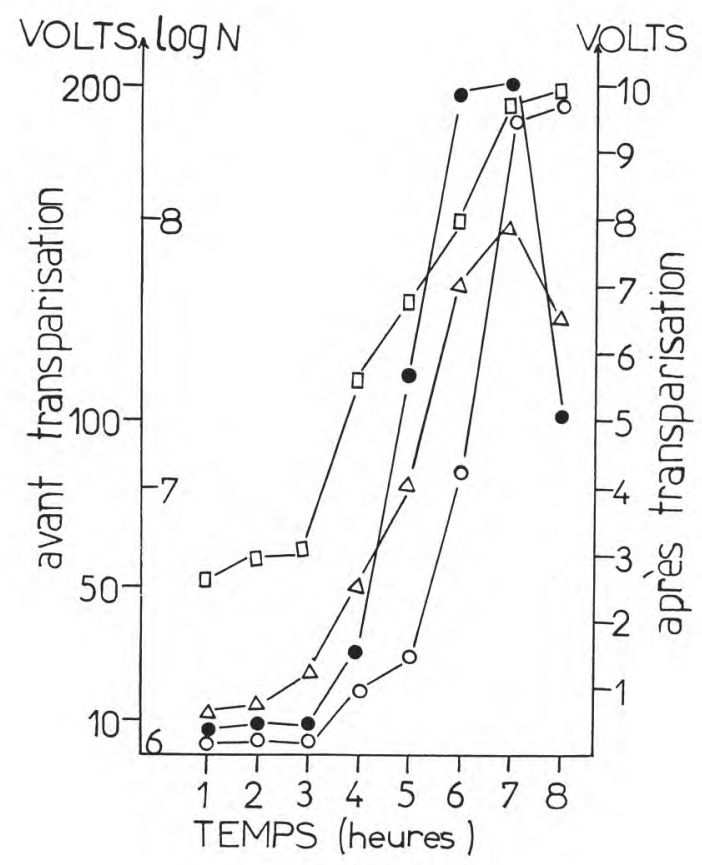

fig. 2

Courbes de croissance de $S$. thermophilus CNRZ 302 dans les milieux TryptoseProtéose-Peptone-Yeast extract (T.P.P.Y.) et lait.

$\square-\square$ Courbe de référence sur milieu T.P.P.Y. (log. $\mathrm{N}=\mathrm{f}$ (Temps).

$\bigcirc-\bigcirc$ Milieu T.P.P.Y. avant transparisation.

- Milieu T.P.P.Y. après transparisation.

$\triangle \longrightarrow$ Lait après transparisation.

Growth curve of S. thermophilus CNRZ 302 in milk and Tryptose-ProteosePeptone-Yeast extract (T.P.P.Y.) medium.

$\square-\square$ Reference curve on T.P.P.Y. medium $(\log N=f$ (Time).

○- $\bigcirc$ T.P.P.Y. medium before transparisation.

- - - T.P.P.Y. medium after transparisation.

$\triangle \longrightarrow$ Milk after transparisation.

parisation. Nous notons comme ci-dessus une perte de sensibilité égale à une puissance de 10 . Les dilutions dans le lait et dans le tampon Tris $0,025 \mathrm{M}$ à $\mathrm{pH} 7,0$ donnent des courbes qui se confondent ; le milieu de dilution n'influe pas sur la réponse enregistrée. 


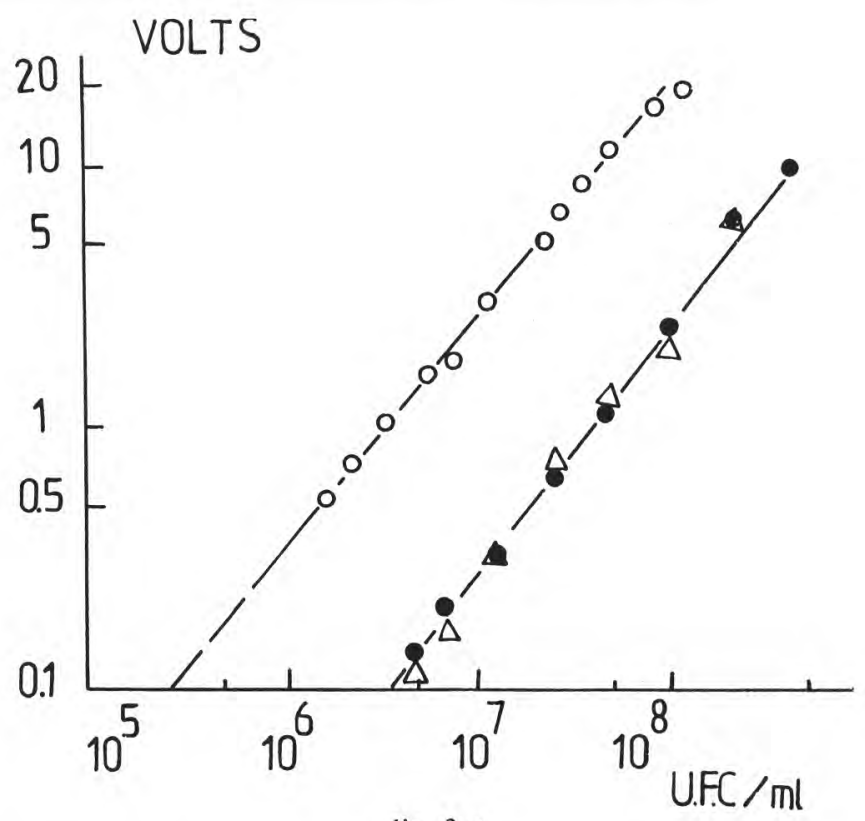

fig. 3 a

Courbe d'étalonnage du néphélémètre laser pour une souche de bactéries Gram négative E. coli (Ga5) dans le tampon Tris $0,025 \mathrm{M}$ à $\mathrm{pH} 7,0$ et dans le lait.

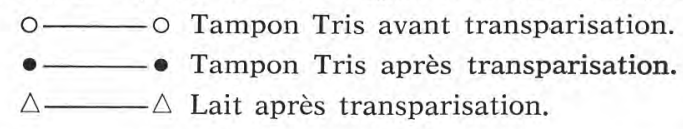

Calibration curve for the laser nephelemeter of a Gram negative bacteria species E. coli (Ga5) in milk and Tris buffer $0.025 \mathrm{M}$ at $\mathrm{pH}$ 7.0.
○— 0 Tris buffer before tranpsarisation.
- - Tris buffer after transparisation.
$\triangle \longrightarrow$ Milk after transparisation.

\section{Application à la courbe de croissance}

Cette étude a été faite sur une souche de Streptococcus thermophilus CNRS 302. Après un ensemencement d'environ $2 \%$ de milieu T.P.P.Y. liquide et de lait à l'aide d'une souche de S. thermophilus CNRZ 302, Rousselot (1982) a pratiqué toutes les heures des prélèvements pour suivre la croissance des bactéries au néphélémètre laser et au spectromètre d'absorption moléculaire. Les courbes de croissance tracées en fonction du temps d'incubation, en tenant compte des dilutions effectuées, montrent qu'avant transparisation, seule la courbe de dilution $1 / 8$ couvre de façon satisfaisante l'échelle de lecture. Après transparisation, il n'est pas nécessaire de faire une dilution 


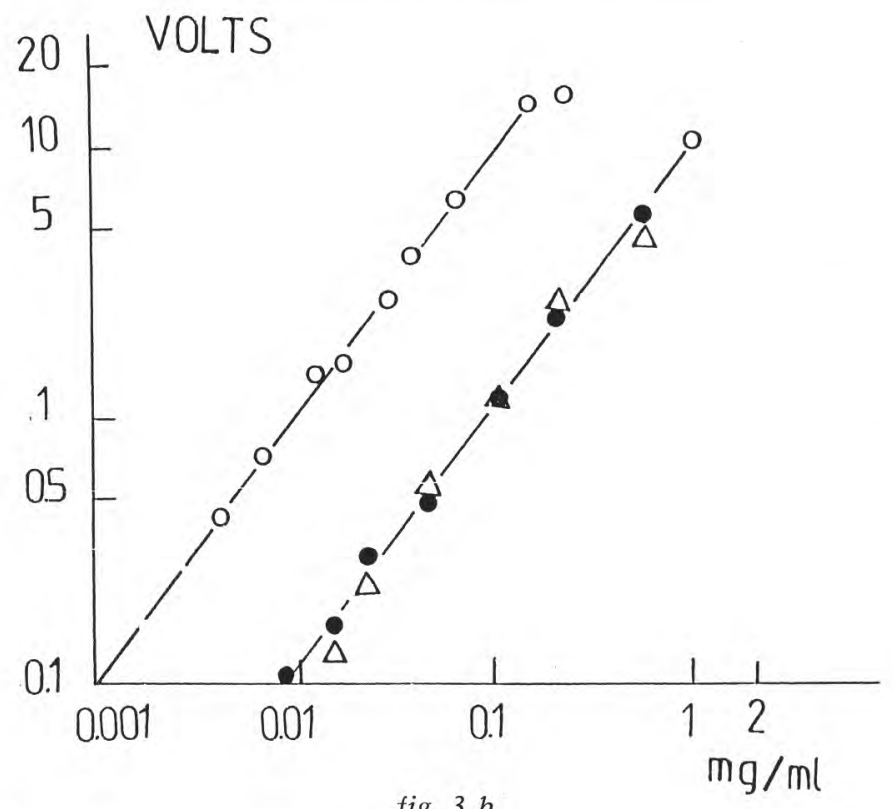

fig. $3 b$

Courbe d'étalonnage du néphélémètre laser pour une souche de bactéries Gram négative E. coli (Ga5) dans le tampon Tris $0,025 \mathrm{M}$ à $\mathrm{pH} 7,0$ et dans le lait.

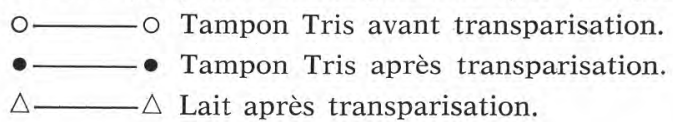

Calibration curve for the laser nephelemeter of a Gram negative bacteria species E. coli (Ga5) in milk and Tris buffer 0.025 $M$ at pH 7.0.

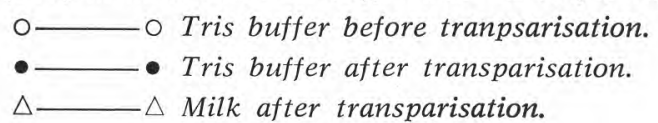

pour avoir une courbe d'allure semblable à celle obtenue avant transparisation (fig. 2).

\section{Essai sur les bactéries Gram négative : étude des différentes souches}

La même expérience que précédemment a été réalisée sur des souches pures de bactéries Gram négative et les résultats obtenus sont semblables à ceux obtenus avec les bactéries Gram positive.

Les figures $3 \mathrm{a}$ et $\mathrm{b}$ nous montrent que les courbes des bactéries Gram négative ont la même allure que celles des Gram positive aussi bien avant qu'après transparisation ; le seuil de détection varie en 


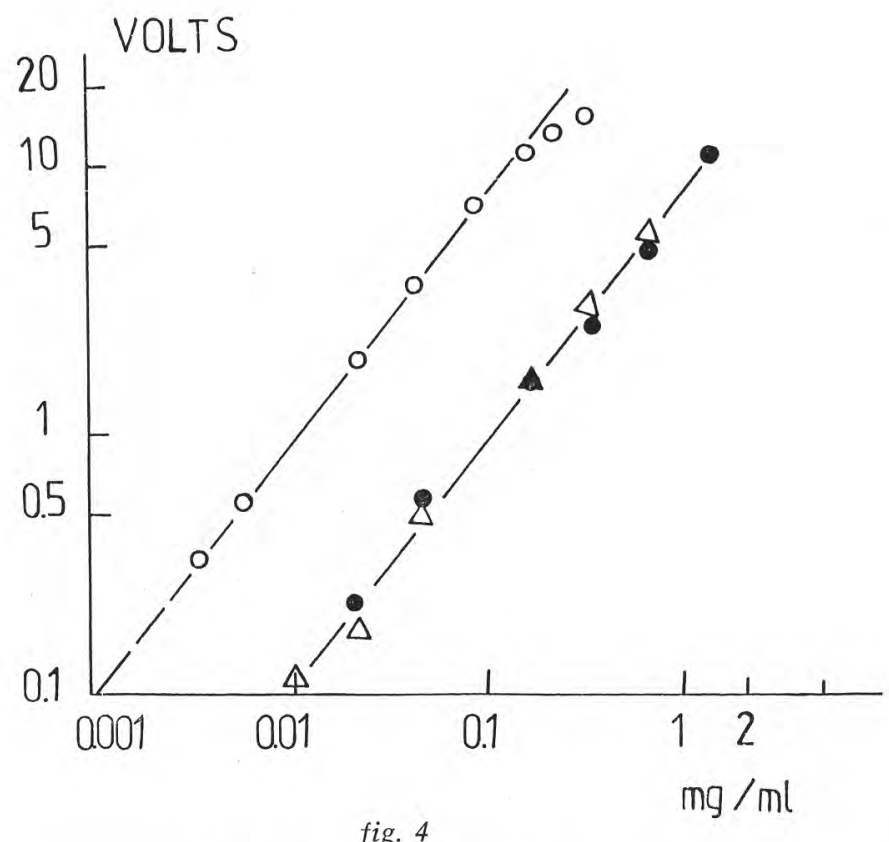

Courbe d'étalonnage du néphélémètre laser pour un mélange de souche de bactéries Gram positive : S. thermophilus CNRZ 302 et Gram négative : E. coli (Ga5) dans le tampon Tris $0,025 \mathrm{M}$ à pH 7,0 et dans le lait.

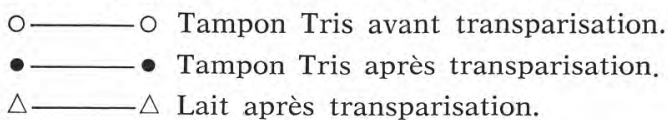

Calibration curve for laser nephelemeter of a mixture of Gram positive : S. thermophilus CNRZ 302 and Gram negative : E. coli (Ga5) bacterium species in milk and Tris buffer $0.025 \mathrm{M}$ at $\mathrm{pH} 7.0$.
O- $\bigcirc$ Tris buffer before transparisation.
- - Tris buffer after transparisation.
$\triangle \longrightarrow$ Milk after transparisation.

fonction de la souche si nous travaillons en nombre de germes mais lorsque nous nous rapportons au poids sec des cellules, ce seuil ne varie pas (fig. $3 \mathrm{a}$ et $3 \mathrm{~b}$ ).

\section{Essai sur les bactéries Gram positive et Gram négative en mélange : étude des différents mélanges}

Les essais sur les souches pures de bactéries Gram positive et Gram négative ayant débouché sur des résultats assez satisfaisants, nous avons pratiqué la même expérience sur des souches de bactéries 


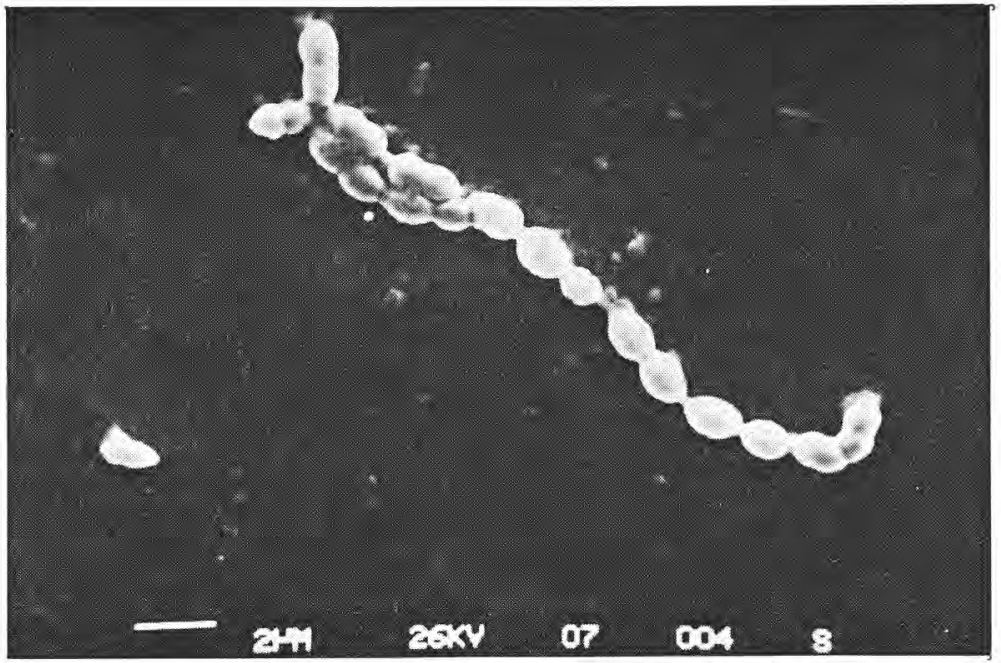

fig. 5 a

Cellules de $S$. thermophilus CNRZ 302 observées au microscope avant et après transparisation.

Avant transparisation.

Cellules en chaînettes (Cocci).

Dimensions : $\varnothing: 0,55 \mu \mathrm{m}$.

$$
\mathrm{L}: 0,71 \mu \mathrm{m} \text {. }
$$

The cells of S. thermophilus CNRZ 302 examined before and after transparisation under a microscope.

Before transparisation.

Cells in assembly line (Cocci).

Dimension : $\varnothing: 0.55 \mu \mathrm{m}$.

$L: 0.71 \mu \mathrm{m}$.

en mélange, essayant ainsi de simuler les conditions existant dans un lait industriel.

Tous nos mélanges de souches ont été effectués, volume à volume, à partir des suspensions-mères des souches pures de bactéries de morphologie identique ou non.

Les résultats obtenus sont identiques à ceux obtenus avec les bactéries Gram positive et négative en souches pures individuelles (fig. 4).

\section{Observation des cellules au microscope}

Avant transparisation

L'observation des cellules en suspension dans le tampon Tris non transparisé a été faite au microscope photonique et électronique à 


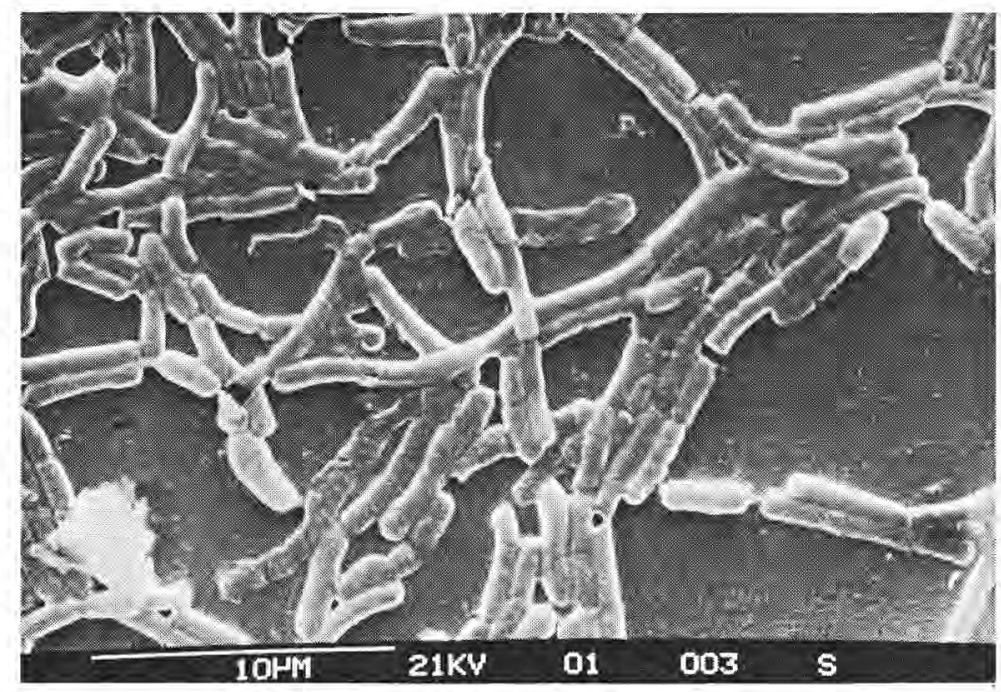

fig. 6 a

Cellules de $B$. Cereus Na5 observées au microscope avant et après transparisation. Avant transparisation.

Cellules en chaînettes ou en amas.

Dimensions : $\varnothing: 0,83 \mu \mathrm{m}$.

$$
\mathrm{L}: 2,7 \mu \mathrm{m} \text {. }
$$

The cells of $B$. Cereus examined before and after transparisation under a microscope.

Before transparisation.

Cells in assembly line or in heap.

Dimension : $\varnothing: 0.83 \mu \mathrm{m}$.

$L: 2.7 \mu \mathrm{m}$.

balayage, en vue de connaître leur morphologie et de vérifier la pureté des souches étudiées (fig. 5a et 6a).

\section{Après transparisation}

Nous avons eu quelques difficultés à observer les cellules en suspension dans le lait transparisé, notamment en microscopie électronique où le mélange dissolvant les constituants colloïdaux du lait masque les cellules. C'est pourquoi toutes nos observations ont été faites sur des cellules en suspension dans le tampon Tris transparisé pendant des intervalles de temps variables.

- Chez les bactéries Gram positive, en travaillant dans les proportions $2 / 1$ i.e. 2 gouttes de suspension-mère de cellules non diluée pour 1 goutte de mélange dissolvant, nous avons constaté que les cellules ne commencent à être lysées qu'au bout d'une trentaine de 


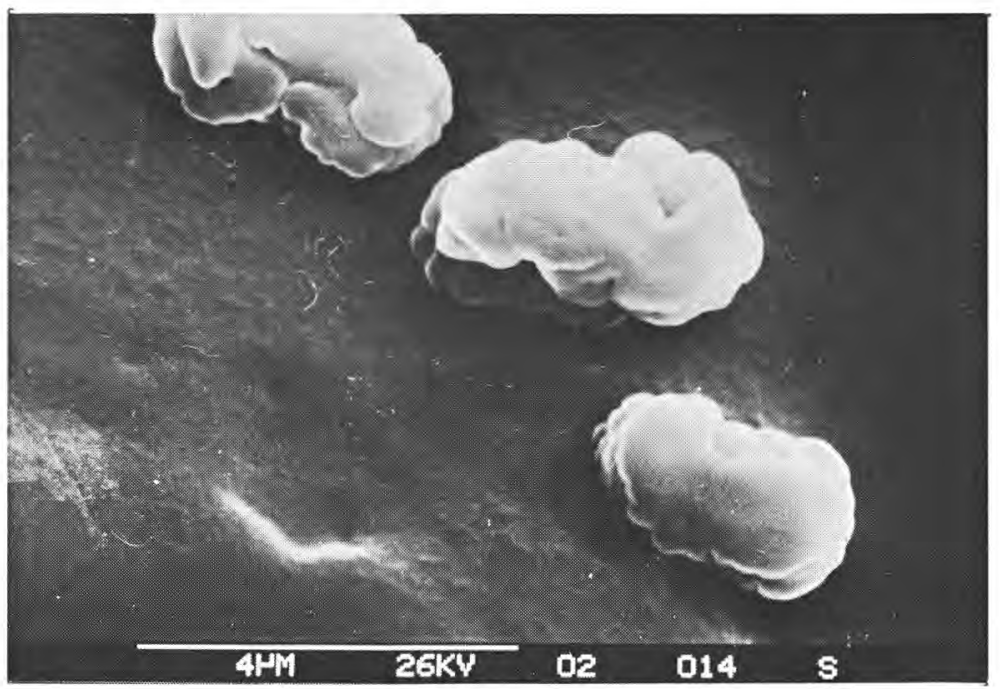

fig. $5 b$

Cellules de $S$. thermophilus CNRZ 302 observées au microscope avant et après transparisation.

Après transparisation.

Cellules très boursoufflées avec contours irréguliers.

Dimensions : $\varnothing: 1,6 \mu \mathrm{m}$.

$$
\mathrm{L}: 3,2 \mu \mathrm{m} \text {. }
$$

The cells of S. thermophilus CNRZ 302 examined before and after transparisation under a microscope.

After transparisation.

Very puffy cells with irregular outline.

Dimension : $\varnothing: 1.6 \mu \mathrm{m}$.

$L: 3.2 \mu \mathrm{m}$.

minutes pour les bacilles et au bout de 45-60 min pour les cocci. Une mesure des dimensions des cellules avant et après transparisation montre que ce phénomène de lyse passe par une phase d'augmentation de volume pendant laquelle les cellules deviennent boursouflées et présentent des contours irréguliers (fig. 5b et 6b).

- Chez les bactéries Gram négative, la lyse est très rapide ; elle est quasi totale $10 \mathrm{~min}$ après l'ajout du mélange dissolvant ; elle est précédée par une phase de modification profonde de la morphologie des cellules, celles-ci devenant très boursouflées avant d'éclater.

- Les résultats obtenus avec les souches en mélange confirment les résultats ci-dessus ; les bactéries Gram négative sont plus rapidement détruites comparées aux bactéries Gram positive. Mais si nous 


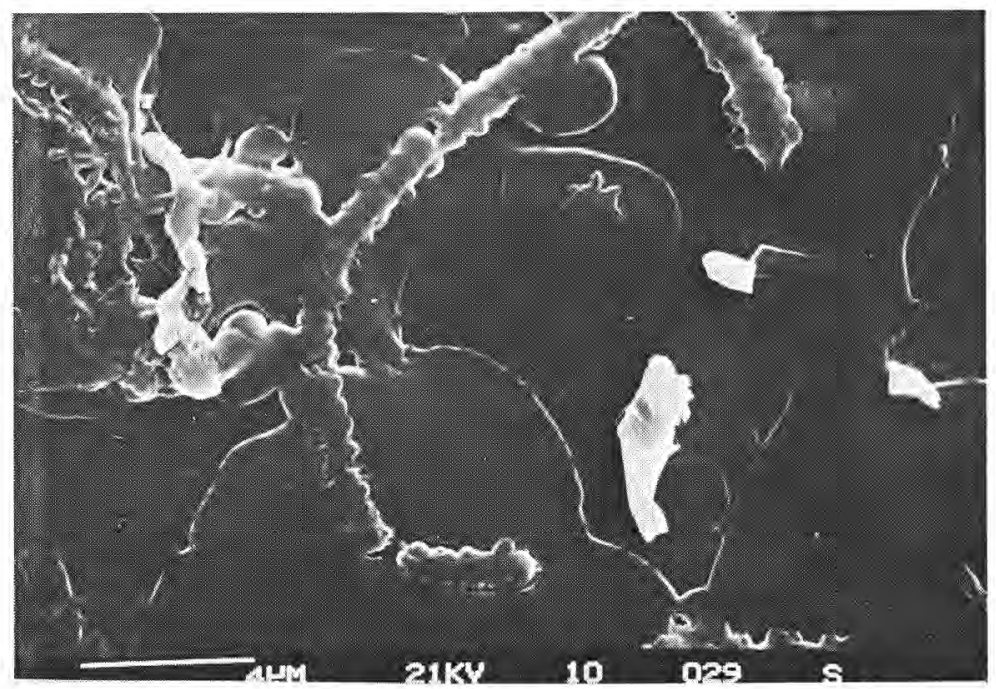

fig. $6 \mathrm{~b}$

Cellules de $B$. Cereus Na5 observées au microscope avant et après transparisation.

Après transparisation.

Cellules boursoufflées à contours dichiquetés.

Dimensions : $\varnothing: 1,6 \mu \mathrm{m}$.

$\mathrm{L}: 5,1 \mu \mathrm{m}$.

The cells of $B$. Cereus examined before and after transparisation under a microscope.

After transparisation.

Puffy cells with shred outline.

Dimension : $\varnothing: 1.6 \mu \mathrm{m}$.

$L: 5.1 \mu \mathrm{m}$.

sommes en présence d'un mélange de Gram positive de morphologie différente telle un coccus et un bacille, c'est le bacille qui est dégradé en premier.

\section{DISCUSSION - CONCLUSION}

La néphélémétrie laser est une technique simple et rapide d'évaluation du nombre de micro-organismes du lait et des produits laitiers. Son large domaine de mesure s'étend de $10^{5}$ à $10^{8}$ U.F.C. $/ \mathrm{ml}$ pour des cellules intactes ; elle constitue donc une technique plus performante que la spectrométrie d'absorption moléculaire dont le seuil inférieur de détection est de $10^{6}$ U.F.C. $/ \mathrm{ml}$. 
Selon le principe même de la néphélémétrie laser, l'intensité de la lumière dispersée mesurée est proportionnelle à la taille et à la forme des particules en suspension. Toutes nos bactéries n'ayant pas la même taille, ni la même forme, l'intensité de la lumière dispersée mesurée ne sera pas la même d'une bactérie à l'autre. Nous n'avons rapporté que deux courbes mais toutes celles que nous avons établies montrent la variation du seuil de détection en fonction des souches lorsque nous travaillons en nombre de germes (U.F.C./ml).

Pour dénombrer rapidement une souche pure, il faut au préalable réaliser une courbe d'étalonnage. Mais pour un lait polymicrobien, il se posera des problèmes puisque toutes les bactéries ne répondent pas de la même manière en néphélémétrie laser. Il serait donc avantageux de travailler en poids sec puisqu'on n'observe pas de variation du seuil de détection quelle que soit la bactérie ; cette pratique n'est, hélas, pas courante chez les microbiologistes.

Le mélange dissolvant $\mathrm{n}$-butylamine/cyclohexanone/triton X-100 présente de nombreux avantages dont celui de ne pas nécessiter une dilution préalable du lait. Il élimine la turbidité naturelle du lait par dissolution complète et rapide de ses différents constituants colloïdaux, permettant ainsi de suivre par une méthode optique la croissance d'une souche de bactéries en milieu liquide et d'évaluer rapidement les micro-organismes présents dans le lait.

La lyse dans le temps des cellules au contact du réactif de transparisation ajouté dans le milieu réactionnel restreint quelque peu le domaine d'application de cette technique de dénombrement.

\section{Bibliographie}

Bosset (J. O.) et Blanc (B. H.) (1977). - Nouvelle méthode simple et économique destinée au dosage de routine des protéines du lait et de quelques produits laitiers. Lait. Rom., 53, 697-699.

Bosset (J. O.), Blanc (B. H.) et Plattner (E.) (1977 a, b). - La dissolution intégrale du lait au moyen de solvants mixtes. I. Etude du mélange dissolvant eau/ hydroxyde de sodium/n-butylamine. II. Etude du mélange dissolvant eau/ hydroxyde de sodium/tétrahydrofurane. Trav. Chim. Aliment. Hyg., 68, 225-239, 504-512.

Bossurt (R.) (1982). - Techniques modernes d'appréciation de la qualité bactériologique du lait. La Tech. Lait., 969, 9-19.

Bouix (M.), Goursaud (J.) et Leveau (J. Y.) (1982). - Essai de " l'Eurotube Roche " appliqué au contrôle microbiologique du lait. Ind. Aliment. Agric., 99 (10), 811-814.

Ciret (P.) (1980), - Les protéines de la coagulation par l'immuno-néphélémétrie à rayon laser. Citée dans document technique de l'Institut Behring.

Gallant (J. D.) (1981). - Microscopie électronique. In Linden et coll. (1981). Techniques d'analyse et de contrôle dans les industries agro-alimentaires, Tome 2, 224-242.

Guinet (R.), Gabriel (S.) et Duforeau (C.) (1979). - Essai d'application de la néphélémétrie laser en bactériologie. Compte rendu groupe d'étude Bendor. 
Linden (G.) and Paquet (D.) (1981). - Alkaline phosphatase activity in transparent milk and cream. J. Dairy Sci., 64, 568-571.

Linden (G.), Humbert (G.), Desnouveaux (R.) et Picard (Jocelyne) (1982). - Application de la dissolution complète du lait à la détermination de quelques activités enzymatiques. Le Lait, 62, 209-219.

Rousselot (Corinne) (1982). - Application de la dissolution intégrale du lait au dénombrement de sa flore mésophile par la néphélémétrie à rayon laser. Rapport de stage. I.U.T. Nancy I.

Süpi (J.), Suhren (G.), Heeschen (W.) und Tolle (A.) (1982). - Die anwendung des limulus-test zur untersuchung ultra hocherhitzter milch und ermittling der bakteriologisch hygienischen wertikeit des verwendeten Rohstoffler. Milchwissenschaft, 37 (6), 341-346. 